\title{
Research on Forming and Polishing Technology Based on Belt Flap Wheel
}

Xing-Wei $\mathbf{S}^{1 *}$, Qin-Yuan $\mathbf{G}^{1}$, He-Ran $Y^{1}$ and Zheng-Qing $Z^{2}$

${ }^{1}$ College of Mechanical Engineering, Shenyang University of Technology, Shenyang, P.R. China

${ }^{2}$ Northern Heavy Industries Group Co, Ltd., 110141, P.R. China

\begin{abstract}
Belt flap wheel is a kind of special-shaped coated abrasives with the advantages of good polishing quality, long life, high efficiency, low cost and so on. It is suitable for polishing various curved surface parts such as shaft, large aperture and large plane. Combined with these polishing characteristics of belt flap wheel, this paper provides a new polishing method of smooth spiral surface forming polishing method based on belt flap wheel with high precision, high efficiency and low cost. This paper analysed the polishing mechanism of spiral curved surface, establishing a mathematical model of screw helical surface. The space contact between the flap wheel and the helical surface was determined. The profile of the flap wheel was designed. The dressing technology of the profile of the belt flap wheel was studied, and a simple dressing test device was set up to complete the finishing test of the flap wheel. The results show that it is feasible to use the belt flap wheel to polish the screw surface.
\end{abstract}

Keywords: Screw; Helical surface; Flap wheel; Forming polishing; Profile; Finishing processing

\section{Introduction}

Screw rotor is the key component of screw machinery. The screw rotor processed by milling technology appears to be scaly knife marks on the spiral surface, it cannot be used directly before application of polishing technology throws the knife to remove. The traditional solution is to use manual polishing or abrasive belt NC polishing [1-3]. The screw profile with the manual polishing is less accurate, it cannot meet the requirements, affects the performance of the screw, moreover, the polishing efficiency is low, the working environment is relatively poor [4]. The abrasive belt NC polishing is limited by the lower life of the belt and needs to replace the belt frequently during polishing, in the polishing of multi-head screw, it is necessary to use a narrow belt to polish the bottom of the screw spiral groove, this greatly reducing the polishing efficiency $[5,6]$.

Belt flap wheel is a kind of polishing tools that collects both of abrasive belt grinding and flexible polishing, it has the advantages of long service life, good polishing quality, high efficiency, good heat dissipation and so on, it can be applied to different occasions due to the flexibility of the blade, in particular, it has special advantages for grinding and polishing the workpiece surface, therefore, the impeller can be well matched with the surface of the workpiece $[7,8]$.

This paper proposed a new method that polishes screw surface with the belt flap wheel, studied the principle of forming and polishing of the flap wheel, determined the profile of the belt flap wheel. It provides a theoretical basis for the following research on the forming and polishing equipment of flap wheel.

\section{Polishing Machining Mechanism of Spiral Surface Analysis}

The main purpose of polishing is to reduce the surface roughness and improve the surface finish quality of the product, further to remove minor defects on the surface of the products, get bright and beautiful surface, or prepare for subsequent surface treatment. Different helical surface screws often correspond to different cross-sectional profile, pitch of screw, helix angle and other parameters. In order to make the helical surface polished by the belt flap wheel on various parts, it is necessary to trim the axial truncation of the belt flap wheel into a shape conforming to the profile of the screw section $[9,10]$ in the process of

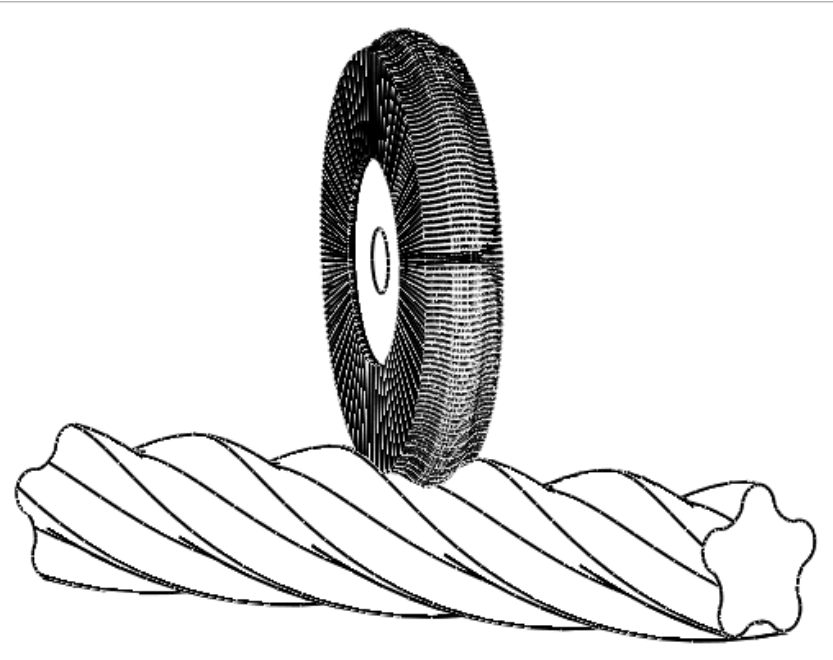

Figure 1: The polishing mechanism of the belt flap wheel.

grinding and polishing, the installation angle of the belt flap wheel is the same as the helix angle of the screw during primary selection, first choice of the center distance of the belt flap wheel and the screw is the sum of the corresponding radius of two workpieces, make the flap wheel rotating around its own axis of the main movement $v_{s}$, the screw does the axial feed movement $Z$ and the rotary feed movement $v$ according to the lead and helix angle, so that the forming and polishing of the screw can be realized in a fixed position by the belt flap wheel, The polishing mechanism is shown in Figure 1.

*Corresponding author: Xing-wei S, College of Mechanical Engineering Shenyang University of Technology, Shenyang-110870, P.R. China, Tel: +86 24 2549 6000; E-mail: sunxingw@126.com

Received October 04, 2017; Accepted November 13, 2017; Published November 16, 2017

Citation: Xing-Wei S, Qin-Yuan G, He-Ran Y, Zheng-Qing Z (2017) Research on Forming and Polishing Technology Based on Belt Flap Wheel. J Appl Mech Eng 6: 294. doi: $10.4172 / 2168-9873.1000294$

Copyright: (c) 2017 Xing-Wei S, et al. This is an open-access article distributed under the terms of the Creative Commons Attribution License, which permits unrestricted use, distribution, and reproduction in any medium, provided the original author and source are credited. 


\section{Profile Design for Contour Machining Tool of Helical Surface}

\section{Coordinate system of polishing flap wheel}

The flap wheel rotates about its own axis while moving along the axis of the rotor, forms the interpolation movement of helical direction with the rotation of the screw rotor. The instantaneous contact between the flap wheel and the rotor is linear contact during polishing; therefore, in order to process the right spiral surface of the rotor, it is necessary to accurately calculate the cross-section profile of the flap wheel. This paper discussed the right-handed screw rotor; the left-handed screw rotor can be studied in a similar manner.

According to the spatial position of the flap wheel and the screw in the process of forming and polishing and the principle of forming and polishing, this paper established the spatial coordinate system as shown in Figure 2, the helical surface of the rotor and the flap wheel is conjugate meshing. In the figure, the coordinate system $S_{1}\left(O_{1}-x_{1} y_{1}\right.$ $z_{1}$ ) is fixed on the screw, the axis $z_{1}$ along the axis of the screw; the coordinate system $S_{2}\left(O_{2}-x_{2} y_{2} z_{2}\right)$ is fixed on the belt flap wheel, the axis $z_{2}$ along the axis of the flap wheel, the axis $x_{2}$ coincides with the axis $x_{1}$ in the screw coordinate system and in the same direction. The shortest distance $O_{1} O_{2}=a$ is the center distance of the meshing pair; the axis $x_{1}$ $\left(x_{2}\right)$ is on the vertical line of the axis $z_{1}$ and the axis $z_{2}$; the corner of the axis $z_{1}$ and the axis $z_{2}$ is $\alpha$, which is the setting angle of the flap wheel. According to the meshing principle, the transformation relationship between the two coordinate systems is:

$$
\left\{\begin{array}{l}
\mathrm{x}_{2}=\mathrm{x}_{1}-\mathrm{a} \\
\mathrm{y}_{2}=\mathrm{y}_{1} \cos \alpha+\mathrm{z}_{1} \sin \alpha \\
\mathrm{z}_{2}=-\mathrm{y}_{1} \sin \alpha+\mathrm{z}_{1} \cos \alpha
\end{array}\right.
$$

Where: $a$ is the center distance, which stands for the shortest distance between the axis of the screw rotor and the rotation axis of the flap wheel; $\alpha$ is the setting angle, which stands for the angle between the axis of the screw rotor and the rotation axis of the flap wheel.

\section{Mathematical model of rotor spiral surface}

The spiral surface of the screw rotor can be seen as its end face, normal curve or the axial sectional curve helically moved along the spiral direction. In the rectangular coordinate system $S_{1}\left(O_{1}-x_{1} y_{1} z_{1}\right)$, let sectional curve of the rotors spiral surface is $\Gamma$, the vector equation can be expressed as:

$$
r_{0}=r_{0}(u)
$$

$u$ is the spatial curve parameter. Let the unit vector of the three

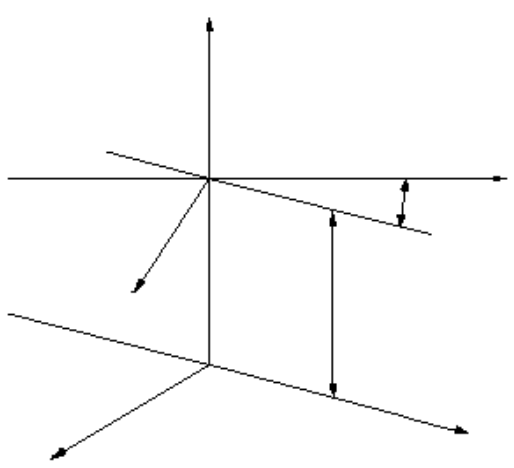

Figure 2: Spatial coordinate system of engagement pairs. coordinate axes $x_{1}, y_{1}, z_{1}$, are respectively expressed as $i, j, k$, so the vector equation of the sectional curve can be expressed as:

$$
r_{0}=r_{0}(u)=x_{0}(u) I+y_{0}(u) j
$$

According to the formula of vector rotation, the equation of the spiral surface of the rotor can be obtained:

$$
r_{0}=r_{0}(u)(k, \theta)^{R}+p \theta k
$$

Where: $\theta$ is the angle parameter, which indicates the angle of the sectional curve $\Gamma$ rotating around the axis $z_{1}$ from the starting position, counter-clockwise rotation is positive; $p$ is the spiral parameter, which indicates the distance of a point on the sectional curve $\Gamma$ moving along the axis $z_{1}$ while the curve $\Gamma$ rotating an unit angle $\theta$, and $\mathrm{p}=p_{z} /(2), p_{z}$ is the lead of the rotor spiral surface. $r_{0}(u)(k, \theta)^{R}$ represents the rotation vector, and the following formula can be obtained:

$$
\boldsymbol{r}_{\mathbf{0}}(\boldsymbol{u})(\boldsymbol{k}, \theta)^{\mathrm{R}}=\cos \theta \boldsymbol{r}_{\boldsymbol{0}}+\sin \theta\left(\boldsymbol{k} \times \boldsymbol{r}_{\mathbf{0}}\right)
$$

Put formula (3) and (5) into (4) and expand it, we can obtain:

$$
\begin{aligned}
& \boldsymbol{r}_{\mathbf{0}}=\left(\mathrm{x}_{0}(\mathrm{u}) \cos \theta-\mathrm{y}_{0}(\mathrm{u}) \sin \theta\right) \boldsymbol{i}+ \\
& \left(\mathrm{x}_{0}(\mathrm{u}) \sin \theta+\mathrm{y}_{0}(\mathrm{u}) \cos \theta\right) \boldsymbol{j}+\mathrm{p} \theta \boldsymbol{k}
\end{aligned}
$$

In the rectangular coordinate system, it can be expressed as:

$$
\left\{\begin{array}{l}
\mathrm{x}_{1}=\mathrm{x}_{0}(\mathrm{u}) \cos \theta-\mathrm{y}_{0}(\mathrm{u}) \sin \theta \\
\mathrm{y}_{1}=\mathrm{x}_{0}(\mathrm{u}) \sin \theta+\mathrm{y}_{0}(\mathrm{u}) \cos \theta \\
\mathrm{z}_{1}=\mathrm{p} \theta
\end{array}\right.
$$

Formula (7) is the mathematical model of the spiral surface of the screw rotor.

\section{Normal vector of screw rotor spiral surface}

Let the normal vector at any point on the rotor spiral surface be $n$, the tangent vector at point $M$ is $\frac{\partial \boldsymbol{r}}{\partial \mathrm{u}}, \frac{\partial \boldsymbol{r}}{\partial \theta}$. Let formula (7) respectively takes the derivative respect $u$ and $\theta$, we can get:

$$
\begin{aligned}
& \left\{\begin{array}{l}
\frac{\partial \mathrm{x}_{1}}{\partial \mathrm{u}}=\mathrm{x}_{0}^{\prime}(\mathrm{u}) \cos \theta-\mathrm{y}_{0}^{\prime}(\mathrm{u}) \sin \theta \\
\frac{\partial \mathrm{y}_{1}}{\partial \mathrm{u}}=\mathrm{x}_{0}^{\prime}(\mathrm{u}) \sin \theta+\mathrm{y}_{0}^{\prime}(\mathrm{u}) \cos \theta \\
\frac{\partial \mathrm{z}_{1}}{\partial \mathrm{u}}=0
\end{array}\right. \\
& \left\{\begin{array}{l}
\frac{\partial \mathrm{x}_{1}}{\partial \theta}=-\mathrm{x}_{0}(\mathrm{u}) \sin \theta-\mathrm{y}_{0}(\mathrm{u}) \cos \theta=-\mathrm{y}_{1} \\
\frac{\partial \mathrm{y}_{1}}{\partial \theta}=\mathrm{x}_{0}(\mathrm{u}) \cos \theta-\mathrm{y}_{0}(\mathrm{u}) \sin \theta=\mathrm{x}_{1} \\
\frac{\partial \mathrm{z}_{1}}{\partial \theta}=\mathrm{p}
\end{array}\right.
\end{aligned}
$$

The normal vector $n$ of the rotor spiral surface can be expressed as:

$$
\boldsymbol{n}=\frac{\partial \boldsymbol{r}}{\partial u} \times \frac{\partial \boldsymbol{r}}{\partial \theta}=\left|\begin{array}{ccc}
\boldsymbol{i} & \boldsymbol{j} & \boldsymbol{k} \\
\frac{\partial x_{1}}{\partial u} & \frac{\partial y_{1}}{\partial u} & \frac{\partial z_{1}}{\partial u} \\
\frac{\partial x_{1}}{\partial \theta} & \frac{\partial y_{1}}{\partial \theta} & \frac{\partial z_{1}}{\partial \theta}
\end{array}\right|=\boldsymbol{n}_{\mathrm{x} 1} \boldsymbol{i}+\boldsymbol{n}_{\mathrm{y} 1} \boldsymbol{j}+\boldsymbol{n}_{\mathrm{z} 1} \boldsymbol{k}
$$




$$
\begin{gathered}
\boldsymbol{n}_{\mathbf{x 1}}=\left|\begin{array}{ll}
\frac{\partial y_{1}}{\partial u} & \frac{\partial z_{1}}{\partial u} \\
\frac{\partial y_{1}}{\partial \theta} & \frac{\partial z_{1}}{\partial \theta}
\end{array}\right|=\frac{\partial y_{1}}{\partial u} \frac{\partial z_{1}}{\partial \theta}-\frac{\partial z_{1}}{\partial u} \frac{\partial y_{1}}{\partial \theta}, \boldsymbol{n}_{\mathbf{y} 1}=\left|\begin{array}{ll}
\frac{\partial z_{1}}{\partial u} & \frac{\partial x_{1}}{\partial u} \\
\frac{\partial z_{1}}{\partial \theta} & \frac{\partial x_{1}}{\partial \theta}
\end{array}\right|=\frac{\partial z_{1}}{\partial u} \frac{\partial x_{1}}{\partial \theta}-\frac{\partial x_{1}}{\partial u} \frac{\partial z_{1}}{\partial \theta} \\
\boldsymbol{n}_{\mathbf{z} 1}=\left|\begin{array}{ll}
\frac{\partial x_{1}}{\partial u} & \frac{\partial y_{1}}{\partial u} \\
\frac{\partial x_{1}}{\partial \theta} & \frac{\partial y_{1}}{\partial \theta}
\end{array}\right|=\frac{\partial x_{1}}{\partial u} \frac{\partial y_{1}}{\partial \theta}-\frac{\partial y_{1}}{\partial u} \frac{\partial x_{1}}{\partial \theta}
\end{gathered}
$$

$n_{x 1}, n_{y 1}, n_{z 1}$ respectively stands for the component of the normal vector of point $M$ in three axes.

\section{The characteristic equation of flap wheel profile}

When using the flap wheel polishing screw rotor groove, a fixed contact line is formed between the rotating surface of the flap wheel and the spiral groove of the rotor. When the contact line revolves around the axis $o_{1} z_{1}$ of the rotor with the same spiral motion as the rotor groove, we can get the rotor spiral surface. When the contact line revolves around the central axis of the flap wheel, the rotary surface of flap wheel is obtained.

If put a cutting plane perpendicular to the axis of flap wheel, then we can obtain a circular intersection $\mathrm{L}$ of the plane and the rotary surface of flap wheel, as is shown in Figure 3.

Let the contact point of the intercept line $L$ and the rotor spiral surface is $M$, then the intercept line $L$ and the rotor spiral surface $M$ should be the existence of the common line, that is the tangent line $T$ through the point $M$ on the intersection line $L$, it must be perpendicular to the normal of the rotor helical surface $n$ at the point $M$, so:

$$
n \bullet \tau=0
$$

Where $\tau$ is the tangent vector on the tangent $T$, it can be expressed as:

$$
\tau=A \times m
$$

Where: $A$ is the direction vector of the rotation axis of flap wheel; $m$ is the radius vector of the point $\mathrm{M}$ on the intersection line $L$ of the flap wheel.

So, the formula (11) can be expressed as;

$$
\mathrm{n} \bullet(A \times m)=0
$$

According formula (13), meshing equation of the flap wheel and screw surface of the screw rotor can be deduced. According to Figure 2 we can know that the direction vector $A$ of the rotation axis $o_{2} z_{2}$ of the flap wheel can be expressed as:

$$
A=(0,-\sin \alpha, \cos \alpha)
$$

The radial vector $m$ of the M-point on the intersection line L can be expressed as:

$$
\mathrm{m}=\left(x_{1}-\mathrm{a}, \mathrm{y}_{1}, \mathrm{z}_{1}\right)
$$

Put formula (14), (15) and (10) into equation (13) and sort it:

$$
\left(\left(x_{1}-a\right) n_{\mathrm{z} 1}-\mathrm{z}_{1} n_{\mathrm{x} 1}\right) \sin \alpha+\left(\left(x_{1}-a\right) n_{\mathrm{y} 1}{ }_{\mathrm{y} 1} n_{x 1}\right) \cos \alpha=0
$$

Simultaneous formula (7) and (15), the meshing line of the spiral surface of the rotor and the flap wheel in the coordinate system $S_{1}\left(O_{1}\right.$ $\left.x_{1} y_{1} z_{1}\right)$, can be worked out, Then, using the transformation relation (1) between coordinate system, we can determine the meshing line in the coordinate system $S_{2}\left(O_{2}-x_{2} y_{2} z_{2}\right)$ of the flap wheel. Set the coordinate $\left(x, y_{i}, z_{i}\right)$ of the corresponding contact point $\mathrm{M}$ in the arbitrary angle of spiral curved surface, the corresponding contact point on the flap wheel can be represented by the following formula:

$$
\left\{\begin{array}{l}
\mathrm{z}_{\mathrm{j}}=-\mathrm{y}_{\mathrm{i}} \sin \alpha+\mathrm{z}_{\mathrm{i}} \cos \alpha \\
\mathrm{r}_{\mathrm{j}}=\sqrt{\mathrm{x}_{\mathrm{j}}^{2}+\mathrm{y}_{\mathrm{j}}^{2}}=\sqrt{\left(\mathrm{x}_{\mathrm{i}}-\mathrm{a}\right)^{2}+\left(\mathrm{y}_{\mathrm{i}} \cos \alpha+\mathrm{z}_{\mathrm{i}} \sin \alpha\right)^{2}}
\end{array}\right.
$$

Formula (14) is the axial section characteristic equation of the flap wheel profile. Here $Z_{j}$ is the half width of the desired point on the flap wheel; $r_{j}$ is the radius of the desired point on the flap wheel.

\section{Design and Calculation for Profile of Belt Flap Wheel}

In the process of designing the profile of the belt flap wheel, the sectional data of spiral curved screws are generally known, the traditional method is to select the appropriate installation angle and the center distance of the belt flap wheel, the general choice of the center distance is $a=(0.7 \sim 1.5) D_{g}, D_{g}$ is the belt diameter of flap wheel, the primary choose of the installation angle is generally $\alpha=90^{\circ}-\beta . \beta$ is the spiral parameter. Based on these parameters, the profile of the belt flap wheel is calculated, if there is a sharp point on the section line, the installation angle should be determined according to the point, and in the actual production process, the installation parameters have to be adjusted according to the technical conditions and test conditions Figure 3. Because of the complex calculation process of profile design of the flap wheel, there is a need of computer programming to simplify the calculation process. The specific design flow is shown in Figure 4.

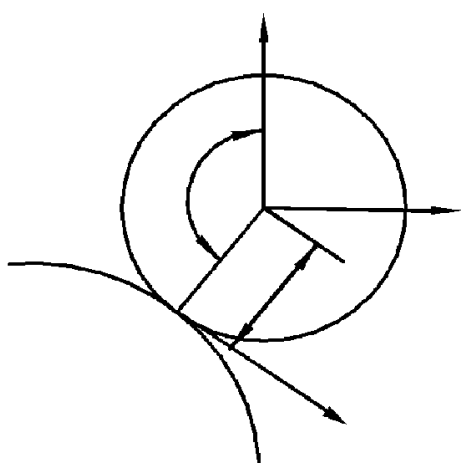

Figure 3: Schematic tangency between flap wheel and spiral curved surface.

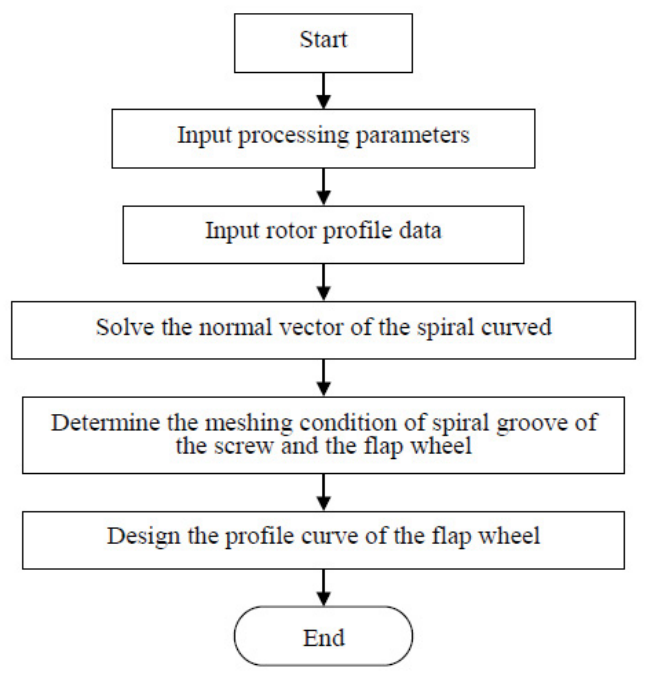

Figure 4: Flow chart for profile design of flap wheel. 


\section{Dressing Technologies for Profile of Belt Flap Wheel}

It is possible to achieve high efficiency, high quality and low cost processing by the use of belt flap wheel surface for forming and polishing with the helical surfaces, however, the market sales of belt flap wheel can not be used directly forming and polishing to the various parameters of the spiral curved surface, most of these flap wheels are cylindrical (Figure 5), It can only be used for polishing simple workpieces such as circular tubes or flat workpieces, to enable it to form and polish the spiral surface, it requires its profile to be able to achieve a good connection with the spiral surface, therefore, it is necessary to finishing the profile of the purchase belt flap wheel (Figure 5).

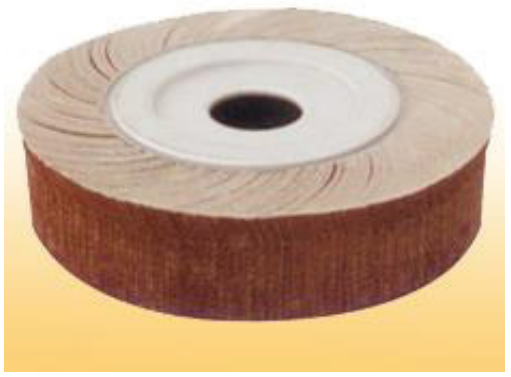

Figure 5: Pre-dressing flap wheel.

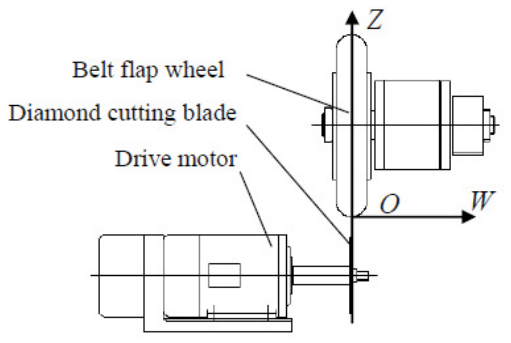

Figure 6: Diagram of belt flap wheel finishing

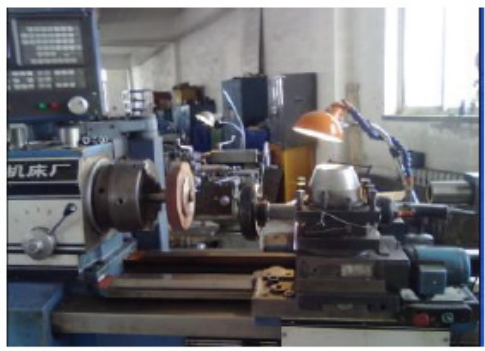

Figure 7: Simple finishing experiment.
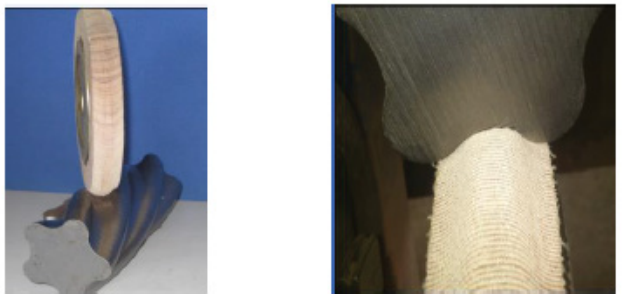

a Meshing principle diagram b partial enlarged diagram

Figure 8: The meshing of the flap wheel and the screw profile.

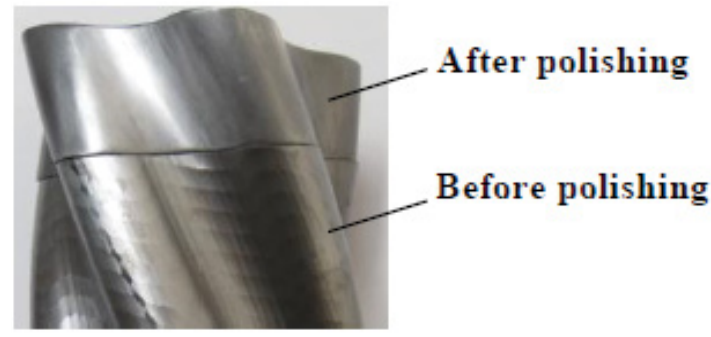

Figure 9: Quality comparison of the screw surface before and after polishing.

Figure 6 shows the finishing of the flap wheel. According to the profile characteristics of the belt flap wheel, we need the belt flap wheel finisher with two-axis linkage function, using a diamond cutting blade to perform an interpolation motion to trim the profile of flap wheel we need. During the finishing process, the diamond cutting blade is driven at high speed by the asynchronous motor, the belt flap wheel also rotates at a normal speed, the rotation center of the diamond cutting blade is controlled by the servo motor to move along a certain locus, the belt flap wheel is fixed through the diamond on the circumference of the diamond cutting blade.

After calculating the profile data of the flap wheel, the flap wheel which will be used to polishing can be obtained by using the flap wheel finishing device. We have used the existing simple experiment equipment to carry on the profile finishing test to the flap wheel shown in Figure 5. In the experiment, we adopt the numerical control lathe of Guangzhou numerical control system to carry on the profile of the flap wheel, The machine tool can achieve the requirements of two axis linkage, the flap a wheel is mounted on a shaft and clamped in the three jaw chuck, the finishing tool is mounted on the angle mill fixed on the lathe slide saddle, the rating power of the angle mill is $2.5 \mathrm{~kW}$, its rated speed is $4000 \mathrm{r} / \mathrm{min}$, as is shown in Figure 7.

The flap wheel trimmed by the test device can achieve profile standard of the rotor screw polishing, the abrasive wear of the surface of flap wheel is good and it is firmly bonded to the substrate, the working surface of flap wheel is uniform and no trimming, the meshing of the flap wheel and screw profile after finishing is shown in Figure 8, the profile of the flap wheel is coincide with the screw surface. Based on the characteristics of flexible polishing of flap wheel, even if there is a small shape error, it will achieve the desired polishing effect.

Finally, the trimming wheel was used to perform a simple polishing experiment on the screw after roughing, the surface quality of the screw before and after polishing is shown in Figure 9, the surface quality of the screw after polishing meet the processing requirements.

\section{Conclusion}

In this paper, design and finishing method for profile of flap wheel are proposed based on the principle of flap wheel forming and polishing processing, by using the simple test equipment, the flap wheel meet the requirements is obtained, and provides a theoretical basis for further study on the forming and polishing for spiral surface of rotor screw, it has important guiding significance for the development of forming machine of screw spiral surface.

\section{References}

1. Wang K, Zang A, Sun $X$ (2010) Study on polishing of screw motor rotors Machine Tool \& Hydraulics 38: 54-56.

2. Jia-Jun W (2011) Theoretical and technical studies on spiral curved surface 
Citation: Xing-Wei S, Qin-Yuan G, He-Ran Y, Zheng-Qing Z (2017) Research on Forming and Polishing Technology Based on Belt Flap Wheel. J Appl Mech Eng 6: 294. doi: 10.4172/2168-9873.1000294

CNC formation polishing. Dissertation of Shenyang University of Technology, Shenyang, China. 2: 1-44.

3. Wei JJ, Wang K, Sun XW (2010) Analysis on the wheel of abrasive belt polishing mechanism to helix curved surface. Computational Intelligence and Natural Computing Proceedings (CINC-P), Wuhan, China. 1: 129-132.

4. Dexiang F (2014) Complex curved surface polishing technology based on fiveaxis machining center. Dissertation of Dalian University of Technology, China.

5. Wei D (2010) Research on control method of screw polishing machine tool. Dissertation of Shenyang University of Technology, Shenyang, China. pp: 10-28.

6. Wang K, Tong-Chun Y, Xing-Wei S (2014) Contact status analysis on the process of dual abrasive belt polishing slender helical curved surface. Mechanical Engineering \& Automation 5:106-108.
7. Qin $L$ (2015) Research on mechanical polishing process for parts with curved surface based on the material removal mechanism. Dissertation of South China University of Technology, China.

8. Lei H, Lingye K, Qiusheng $Y(2010)$ Research on polishing process of mould with abrasive belt wheel. Mechanical \& Electrical Engineering Technology 39: 109-112.

9. Zhengqing $Z$ (2013) Study of forming polishing technology based on smooth spiral curved surface. Dissertation of Shenyang University of Technology, Shenyang, China.

10. Xingwei S, Zhengqing Z, Ke W (2014) Profile designing and finishing method for flap wheel applied in shaping and polishing of spiral curved surface. Dissertation of Shenyang University of Technology, Shenyang, China. 36: 176-181. 